\title{
Community Multimedia Cards: CoMu Cards
}

\author{
Heiko Pfeffer, Steffen Krüssel, Stephan Steglich \\ Fraunhofer Institute for Open Communication Systems (FOKUS) \\ Kaiserin-Augusta-Allee 31 \\ 10589 Berlin, Germany \\ \{heiko.pfeffer|steffen.kruessel|stephan.steglich\}@fokus.fraunhofer.de
}

\begin{abstract}
Within the last years, the Web has developed into a content platform featuring rich multimedia content, dynamic establishments of communities and a continuously increasing importance of interaction and communication. This trend reveals new challenges when considering the increasing mobility of users implied by the usage of portable devices such as PDAs and Multimedia Players.

Within this paper, we present an application for managing, extending and sharing multimedia content within user communities. Here, special emphasis is put on the abstraction from the underlying network topology. Thus, the way specific functionality of the application is realized is dynamically decided with regard to user's preferences and the current network topology.
\end{abstract}

\section{Keywords}

Service Composition, Multimedia, Widgets

\section{INTRODUCTION}

Nowadays, the organization and presentation of multimedia content such as photos, videos, and music is a key requirement for many media players, featuring the management of content on top of standard play-back functionalities. File-sharing tools on the other hand have been developed in order to share this content among users. Due to the lasting triumphal procession of the Web2.0, multimedia content has become even more present in peoples' everyday lives. However, the Web is developing more and more towards a hybrid structure, consisting of devices that are connected by infrastructure components such as servers, and mobile devices spontaneously establishing ephemeral connections.

Within this paper, we introduce a multimedia community application, together with its related architecture. Here, we aim at unifying the three above mentioned challenges of content management, content sharing within communities of users, and mobility within the future Web.

The Community Multimedia Cards provide a user interface for managing their content, while featuring search mechanisms that render the current underlying infrastructure transparent to the user. This feature is achieved by establishing unified interfaces

Permission to make digital or hard copies of all or part of this work for personal or classroom use is granted without fee provided that copies are not made or distributed for profit or commercial advantage and that copies bear this notice and the full citation on the first page. To copy otherwise, or republish, to post on servers or to redistribute to lists, requires prior specific permission and/or a fee.

MobiQuitous 2008, July 21-25, 2008, Dublin, Ireland.

Copyright $\odot$ 2008 ICST ISBN 978-963-9799-27-1 for different kinds of functionality, which allow service compositions to capture the actual processing of the users' request.

\section{State OF The Art}

One of today's problems is the management of content due to the vast amount of data existing. A system should provide structures to be useful for users. Local content management is mostly covered by file systems, such as Apple's Hierarchical File System (HFS) and Microsoft's New Technology File System (NTFS). Current file systems organize files into treestructures. However, content can be also managed with the help of tags [4] as well as semantic descriptions [5]. Beside these local file based solutions content management systems [6] provide additional functionality in the Web, such as collaborative manipulation and secure data management.

Thus, structured content has to be transported to different endpoints for high availability. While many platforms are based on fixed infrastructures, others establish Peer-to-Peer access, but are usually bound to central entrance points as well.

\section{CoMu Architecture}

The main goal of the CoMu application is to provide a simple interface for representing, managing and sharing multimedia content. Here, the underlying network topology should remain hidden for mobile users when searching new content.

Within the CoMu application, a CoMu card is considered as a mashup of multimedia content of a certain musician or band. Here, the content can reach from videos, music files, and images to RSS feeds and blog entries. Beside the organization and management of multimedia content in a trading card style, the $\mathrm{CoMu}$ application provides a unified search interface for sharing $\mathrm{CoMu}$ Cards within a community by providing means to either search new CoMu Cards or to extend existing ones by additional multimedia content.

In order to abstract from the underlying network topology when searching for new content, we distinguish three types of content depending on how the data can be accessed. First, the content may be present on the user's own device, so-called local content. Second, the content can be located on a device of a user who is in short connection range so that the files can be accessed in an ad hoc style; this type of content is referred to as community content. Third, the data may be available within the $\mathrm{Web}$, thus an internet connection is required to access this socalled Web content.

All these content types can be accessed through a unified search interface. Thereby, the available connections to other community members or the Web appear hidden for the user. Through an options menu, preferences can be set defining which access styles and types of content are preferred. Since the access 
of the different kinds of content entails the invocation of different access methods to services retrieving the content, each search request is mapped to a service composition whose single services are dynamically bound.

Thus, when searching content for a specific artist, three major steps are performed:

1. checking the available connections and user preferences with regard to access methods and desired type of content,

2. binding services to the abstract service composition that meets the user's preferences with regard to their access method and are able to retrieve parts of the desired content,

3. executing of the bounded service composition and integrating the resulting content into the according $\mathrm{CoMu}$ Cards.

Notably, search results do not return the actual content itself, but meta-data describing it. This meta-data encompasses some basic information of the specific content in a tag cloud fashion; for instance, for music files this meta-data can be build by mp3s' ID3 tags. Within the CoMu Card, content is then presented with regard to its availability. Local content is marked as available since the content is present on the own device. Search results stemming from community members are marked as temporally available, thus, can be played back as long as the member stays within connection range. In case the connection to the user breaks, the content entry is removed from the CoMu Card. Content that was retrieved from the Web is marked as available on demand; indeed, the content is not locally available and can thus not be played back without restrictions, but it is assumed that the content can be accessed as soon as a connection to the Web is present.

When playing back content that is not available locally the file is streamed to the requesting user. Optionally, the user may chose to download a file explicitly in order to make it locally available.

Thus, by hiding retrieval methods for content behind a unified search interface, a simple access and extension method for multimedia content is provided. This level of abstraction is achieved by a dynamic binding of services to a service composition plan controlling the cooperation of the single services.

\section{Realization}

The realization is based upon Eclipse's Standard Widget Toolkit (SWT) for an easy integration within the target Windows Mobile platform. Since SWT requires a Java 1.4 virtual machine, IMBs J9 is used.

The core component of the $\mathrm{CoMu}$ application is the search service that offers a uniform interface to a metadata search and different content providers. While the search engine delivers metadata about content, the content provider supplies this content. Each datum is available via REST (Representational State Transfer) with the help of RestAC [1], an open-source framework for the simplification of REST interactions. The search service provides a link to the actual resource (content).

While only one interface has to be addressed by the application developer, the search component distributes the search to different endpoints. A local and remote search for the appropriate content is provided, whereas the remote search is identical to the local search, but on remote devices. Furthermore, an additional function has been integrated to request external Web Services.

Thus, the whole search service is designed modular and can be combined freely to meet the user's request. Therefore, different media types and concrete search services (local, remote, Web Service) can be contacted.

\section{Conclusion AND OUTlOOK}

Within this paper, we developed an application for sharing and managing multimedia content within mobile user communities. Therefore, we discussed core principles of the underlying architecture, i.e. the unification of interfaces and dynamic service composition bindings, which enable the abstraction from the underlying network topology.

Within future work, evaluation techniques for both the underlying service compositions and the respectively retrieved content will be integrated within the CoMu application. The work presented in [2] describes a lightweight model to represent non-functional properties of service compositions by means of fuzzy membership functions. We will use the same mechanism to model the quality of the retrieved content. That way, the performance of a service composition can be pondered against the quality of the output it generates. The according evaluation technique will base on a fast fuzzy multiplication algorithms presented in [3].

\section{REFERENCES}

[1] Fraunhofer Institute for Open Communication Systems (FOKUS) projects/restac/

https://developer.berlios.de/projects/restac/

[2] H. Pfeffer, S. Krüssel, and S. Steglich. Fuzzy Service Composition Evaluation in Distributed Environments. Submitted to the International Conference on Advances in Human-oriented and Personalized Mechanisms, Technologies, and Services 2008 (I-CENTRIC 2008).

[3] S. Krüssel, H. Pfeffer, and S. Steglich. Fuzzy Modeling of Resource Consumption for Service Composition Evaluation. Submitted to the Second IEEE International Conference on Self-Adaptive and Self-Organizing Systems 2008 (SASO 2008).

[4] ID3. http://www.id3.org/. 2008

[5] Powerset. http://www.powerset.com/. 2008

[6] Typo3. http://typo3.org/. 2008 